\title{
Electrocatalytic Activity of Galvanostatically Deposited Ni thin Films for Methanol Electrooxidation
}

\author{
Maria Paula Salinas-Quezada ${ }^{a}$, D. Alfonso Crespo-Yapur ${ }^{a}$, Abraham Cano-Marquez $^{b}$, $^{-}$ \\ Marcelo Videa ${ }^{a, *}$ \\ Department of Chemistry, School of Engineering and Sciences, \\ Tecnologico de Monterrey, Av. E. Garza Sada 2501 Sur, Monterrey, NL 64849, Mexico \\ Centro de Investigacion en Materiales Avanzados, S. C. (CIMAV), Unidad Monterrey, \\ Alianza Norte 202, 66600 Apodaca, Nuevo Leon, Mexico
}

\begin{abstract}
Nickel and nickel-based nanomaterials are an attractive choice to replace noble metals as electrocatalyst in alkaline direct alcohol fuel cells (DAFCs) owing to their lower cost and suitable electrocatalytic activity. Among the different synthetic methods available for the production of nanostructured materials, galvanostatic electrodeposition offers a fast and simple means of fabricating active electrodes. Therefore, thin-layers of nickel were electrodeposited onto polycrystalline gold electrodes using constant current pulses from an electrolyte containing $50 \mathrm{mM} \mathrm{NiSO}$. The electrocatalytic properties of the nickel nanostructures in alkaline medium, in which the catalytic species $\mathrm{NiOOH}$ is formed, were evaluated through cyclic voltammetry in $0.5 \mathrm{M}$ methanol $+1 \mathrm{M} \mathrm{KOH}$. The effects of current density pulse and the presence of sulfate or chloride anions in the supporting electrolyte on the electrocatalytic activity of the deposits were studied using $\mathrm{Ni}(\mathrm{OH})_{2}$ surface concentration, $\Gamma$, and electrocatalytic intensity, $E I$, as performance parameters. It was found that highest electrolcatalytic activities were obtained when using current densities pulses close to $4.0 \mathrm{~mA} \mathrm{~cm}^{-2}$ in the presence of sulfates. It was found that the presence of sulfates leads to a strong correlation between the electrocatalytic activity for the oxidation of methanol and the surface concentration of $\mathrm{Ni}(\mathrm{OH})_{2}$.

Keywords: Nickel nanostructuresgalvanic electrodeposition, Methanol oxidation reaction, direct alcohol fuel cells.
\end{abstract}

*corresponding author. mvidea@itesm.mx 


\section{Introduction}

Direct alcohol fuel cells (DAFC) are strong candidates to replace other power sources in automobiles and portable devices [1]. This is mainly due to the fact that the mass energy density of low molecular weight alcohols is comparable to that of gasoline $\left(5-8 \mathrm{~kW} \mathrm{~h} \mathrm{~kg}^{-1}\right.$ vs. $\left.12 \mathrm{~kW} \mathrm{~h} \mathrm{~kg}^{-1}\right)$ [2]. Furthermore, liquid fuels are easier to transport, handle and store. While several studies have focused on the development of acidic DAFC [3], their alkaline counterparts (ADAFC) exhibit some attractive properties: alkaline media facilitates the 4-electron transfer for the oxygen reduction reaction (ORR) since no specific chemical interactions between the catalyst and $\mathrm{O}_{2}$ or $\mathrm{O}_{2}^{-}$are required [4], the oxidation of alcohols, like methanol on $\mathrm{Pt}$ and $\mathrm{PtRu}[5,6]$, can yield higher currents at low overpotentials; the formation of high valence oxide species, which act as catalyst for alcohol oxidation, is favored in alkaline media [7]. Additionally, the development of alkaline anion-exchange membranes has mitigated some of ADAFCs problems like alcohol cross-over, corrosion and carbonate precipitation [8].

Nickel has shown good catalytic activity for alcohol oxidation in alkaline media, compared with silver and cobalt [9]. This is due to the formation of $\mathrm{NiOOH}$, which has unpaired $d$-electrons and empty $d$-orbitals available for bond formation with adsorbed species or redox intermediates [10,11]. Different Ni-nanostructured morphologies, such as nanoparticles [12], ultra-thin sheets [13], and nanoflakes [14] have been tested in efforts to increase the catalyst surface area and to improve their intrinsic catalytic properties. The synthesis of these materials usually involve several complicated steps, like atomic layer deposition [15], ion implantation [16] or electroless deposition [17], contrary to electrodeposition that can be carried out in a single step on the substrate. Previous work by our group proved that ensembles of Ni nanoparticles, with narrow size distribution, can be obtained on ITO electrodes by applying short constant current pulses in a borate-free electrolyte [18]. Galvanostatic electrodeposition offers great control on nanoparticle size through the selection of intensity and duration of the current density pulses, and it can be easily scaled for mass production.

In this work, nickel thin-films electrodeposited onto gold electrodes of well characterized surface area were produced by short galvanostatic pulses from a borate-free electrolyte. The electrodeposition current density pulse, which imposes the electrocrystallization kinetics of nickel, and the presence of sulfate or chloride anions in the supporting electrolyte, were selected as experimental parameters to influence the electrocatalytic activity of the nickel electrodeposits. The $\mathrm{Ni}(\mathrm{OH})_{2}$ surface concentration, $\Gamma$, and electrocatalytic intensity, 
$E I$, were used to evaluate the electrocatalytic properties of the deposits obtained. It was found that highest electrolcatalytic activities were obtained when using current densities pulses close to $4.0 \mathrm{~mA} \mathrm{~cm}^{-2}$ in the presence of sulfates. It was found that the presence of sulfates leads to a strong correlation between the electrocatalytic activity for the oxidation of methanol and the surface concentration of $\mathrm{NiOOH}$ produced during the oxidation of $\mathrm{Ni}(\mathrm{OH})_{2}$.

\section{Experimental Methods}

All glassware was cleaned by soaking it in $1 \mathrm{M} \mathrm{KOH}$ overnight and rinsing with deionized water (EASYpure II $18.2 \mathrm{M} \Omega \mathrm{cm}^{-2}$ ). The electrochemical experiments were carried in a single compartment three-electrode cell with a $\mathrm{Pt}$ spiral as counter electrode. The working electrode was an Au wire (Aldrich, $99.99 \%$ ) with a diameter of $0.5 \mathrm{~mm}$ embedded in epoxy resin effectively exposing the cross section with an average area of $6.0 \pm 0.2 \times 10^{3} \mathrm{~cm}^{2}$. In acidic or neutral media a $\mathrm{Hg} \mid \mathrm{Hg}_{2} \mathrm{SO}_{4}$ sat. $\mathrm{K}_{2} \mathrm{SO}_{4}$ reference electrode (MMSE) was used while for experiments in alkaline electrolytes a $\mathrm{Ag} \mid \mathrm{AgCl}$ sat. $\mathrm{KCl}$ reference electrode was selected. Before the electrodeposition experiments, the gold electrode was polished using alumina of smaller particles size $(1,0.3$ and $0.1 \mu \mathrm{m})$ to achieve a mirror finish with a known and reproducible surface area. Then, the electrode was cleaned in an ultrasonic bath using acetone, isopropanol, ethanol, and deionized water. Electrochemical cleaning was carried by cycling the electrode in $50 \mathrm{mM} \mathrm{H}_{2} \mathrm{SO}_{4}$ (Fermont trace ppb) between -0.7 and $1.06 \mathrm{~V}$ vs. MMSE at a scan rate of $100 \mathrm{mV} \mathrm{s}^{-1}$ until a stable voltammogram was obtained. Following this, the scan rate was changed to $50 \mathrm{mV} \mathrm{s}^{-1}$ and the resulting voltammograms were recorded to compute the electrode surface area using the charge for the reduction of the oxide monolayer, following the methodology described by Lukaszewski et al. [19].

\subsection{Electrochemical Deposition}

Following the electrochemical cleaning, the electrolyte was replaced by a plating solution. This solution contained $50 \mathrm{mM} \mathrm{NiSO}_{4}$ (Sigma Aldrich 99\%) and $0.5 \mathrm{M}$ of a supporting electrolyte that was either $\mathrm{Na}_{2} \mathrm{SO}_{4}$ (CTR Scientific 99.6\%) or $\mathrm{NaCl}$ (CTR Scientific 99.9\%) to investigate the effect of the anions in the electrolytic bath. The Ni deposits were generated by applying a single two-second cathodic current pulse through the working electrode immersed in the electrolytic bath. The cathodic current densities used were 
$1.5,2.2,3.5,4.0,5.0,6.0,8.0$ and $10 \mathrm{~mA} \mathrm{~cm}^{-2}$. The applied current was calculated using the electrode surface area calculated as mentioned above.

\subsection{Oxidation of the nickel deposits}

Once nickel was deposited on the working electrode this was transferred into a $1 \mathrm{M} \mathrm{KOH} \mathrm{(Fermont)} \mathrm{solution} \mathrm{and} \mathrm{subjected} \mathrm{to} 30$ cycles at $50 \mathrm{mV} \mathrm{s}^{-1}$ from -0.4 to $0.48 \mathrm{~V}$ vs. $\mathrm{Ag} \mid \mathrm{AgCl}$. This was done to oxidize the $\mathrm{Ni}(\mathrm{OH})_{2}$ and to promote the formation of $\mathrm{NiOOH}$.

\subsection{Methanol electrooxidation}

The electrocatalytic activity of the deposits obtained was tested by cyclic voltammetry in $0.5 \mathrm{M}$ Methanol $+1 \mathrm{M} \mathrm{KOH}$ at $50 \mathrm{mV} \mathrm{s}^{-1}$ from -0.4 to 0.75 $\mathrm{V}$ vs. $\mathrm{Ag} \mid \mathrm{AgCl}$.

\subsection{Morphology characterization}

Nickel was electrodeposited during five seconds on the surface of a gold evaporated quartz crystal (AMTEK Scientific Instruments) at $3.5 \mathrm{~mA} \mathrm{~cm}{ }^{-2}$, using the same electrochemical cleaning and plating solutions mentioned above. The resulting deposits were examined by scanning electron microscopy (SEM) (Nova NanoSEM 200, FEI, Japan) equipped with EDX analysis (INCA XSight, OXFORD instruments, England) to determine their chemical composition.

\section{Results and Discussion}

Figure 1 depicts the typical behavior of the working electrode potential during a galvanostatic electrodeposition experiment, in which four regimes can be distinguished. After the application of the current pulse, in the first regime, the electrode experiences a potential drop, reaching values more negative than the onset for Hydrogen Evolution Reaction (HER) on gold $(-0.70 \mathrm{~V}$ vs. MMSE from cyclic voltammetry measurements) and the reversible potential for nickel reduction ( $-0.928 \mathrm{~V}$ vs. MMSE, calculated from Nernst equation). During the next regime (II), the potential shows a slow recovery of ca. $150 \mathrm{mV}$ to then follow a dramatic drop in voltage (regime III) and then a gradual polarization towards more negative potentials in regime IV. 


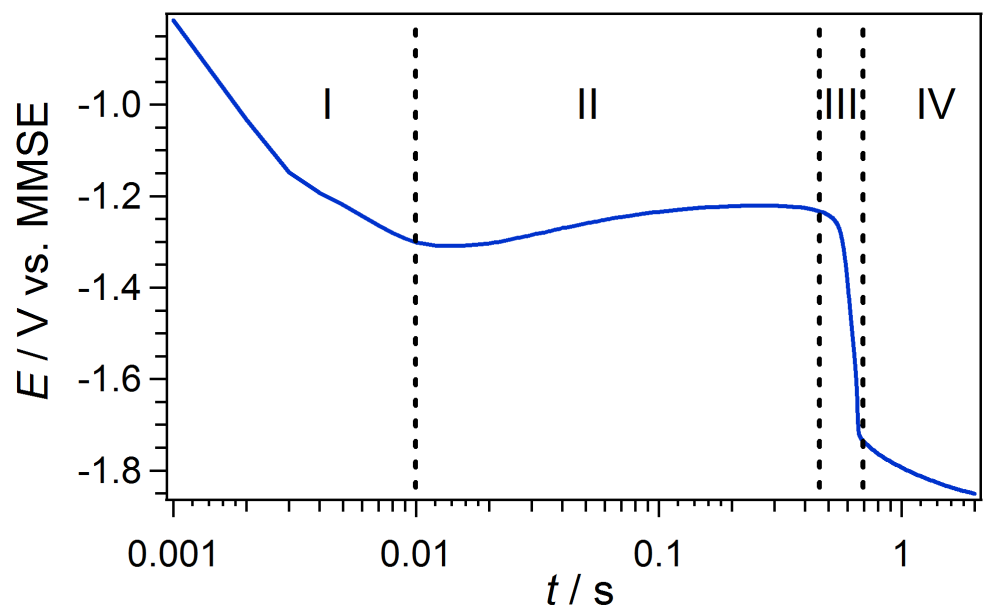

Figure 1: Typical potential transient recorded during a galvanostatic deposition of $\mathrm{Ni}$ on a clean $\mathrm{Au}$ electrode from a $50 \mathrm{mM} \mathrm{NiSO}{ }_{4}+500 \mathrm{mM} \mathrm{Na} 2 \mathrm{SO}_{4}$ plating bath. Applied current density: $8.0 \mathrm{~mA} \mathrm{~cm}^{-2}$. Notice that logarithmic time scale is used, allowing a better inspection of the processes during the electrode polarization at different time scales.

As it can be confirmed from Figures $2 \mathrm{a}$ and $2 \mathrm{~b}$, for sulfate and chloride rich electrolytes, respectively, the minimum potential reached during the first regime becomes more negative and it is attained at shorter times as the applied current density is increased, ranging from $-1.291 \mathrm{~V}$ to $-1.373 \mathrm{~V}$. According to the results reported by Lachenwitzer et al. [20] from Electrochemical Quartz Crystal Microbalance (EQCM) measurements during nickel deposition through cyclic voltammetry, in the range between -0.799 and $-1.099 \mathrm{~V}$ vs. MMSE a cationic species is adsorbed on the working electrode. Thus, the behavior of regime I, the first 10 to $100 \mathrm{~ms}$ depending on the applied current density, can be attributed to the adsorption of the $\mathrm{Ni}^{2+}$ complex on the gold surface and its reduction to $\mathrm{Ni}^{0}[21,22]$. The range of minima reached is also below the critical potential necessary for the formation of nickel nanoparticles on ITO in an electrolyte of similar composition, $-1.243 \mathrm{~V}$ vs. MMSE, indicating that $\mathrm{Ni}$ nuclei are formed on the Au surface in this regime [18].

As it can be seen in Figure2a, for the experiments performed in the sulfate bath, the potential curve in regime II follows similar values regardless of the applied current. This behavior is also observed in the chloride bath (Figure $2 \mathrm{~b})$. While it is likely that this stage corresponds to the growth of the nuclei formed in regime I, the occurrence of parallel processes such as hydrogen evolution reaction (HER) on the surfaces of $\mathrm{Au}$ and the deposited $\mathrm{Ni}$, the 

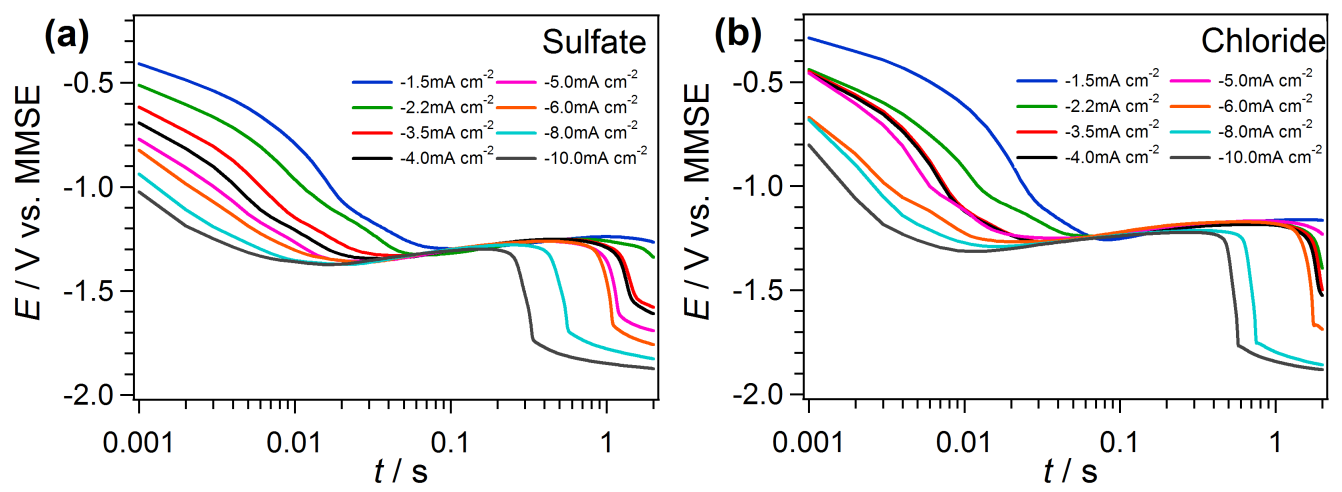

Figure 2: Potential transients recorded during a galvanostatic deposition of $\mathrm{Ni}$ on a clean $\mathrm{Au}$ electrode at different applied currents pulses in different plating baths: (a) $50 \mathrm{mM} \mathrm{NiSO}_{4}+500 \mathrm{mM} \mathrm{Na}_{2} \mathrm{SO}_{4}$ and (b) $50 \mathrm{mM} \mathrm{NiSO}_{4}+$ $500 \mathrm{mM} \mathrm{NaCl}$.

formation and growth of new Ni deposits, and their contributing to a currentindependent polarization should not be discarded. For current pulses larger than $2 \mathrm{~mA} \mathrm{~cm}^{-2}$, a clear second potential drop followed by a slower potential decay can be observed. These regimes, III and IV respectively, indicate a transition to new electrochemical processes once nickel reduction on gold becomes unable to supply the current demand imposed by the galvanostat. Commonly this behavior occurs due to diffusion limitation of the electroactive species or quenching of the electrodeposition process by an adsorption process [23, 24, 25]. Vampaemel et al. [24] observed similar potential curves on their galvanostatic deposition experiments, which were carried out using an electrolyte containing $5-30 \mathrm{mM} \mathrm{NiCl}_{2}, 100 \mathrm{mM} \mathrm{KCl}$ at $p \mathrm{H}=3$. They assigned the second potential drop to the beginning of $\mathrm{H}_{2} \mathrm{O}$ reduction, which was backed by their Sand equation calculations. According to their work, HER changes the local $p \mathrm{H}$ favoring the formation of $\mathrm{Ni}(\mathrm{OH})_{2}$, blocking the deposition process. Figures $2 \mathrm{a}$ and $2 \mathrm{~b}$ show how the onset of regime III shifts to shorter times as the applied current intensity is increased, which is to be expected for a diffusion limited process. Similarly, Wang et al. [25] showed that at high overpotentials, $\mathrm{Ni}$ electrodeposition on gold is stopped by the formation of $\mathrm{Ni}(\mathrm{OH})_{2}$, resulting in a $\mathrm{Ni}$ monolayer. In the presence of chlorides, the electrode potential undergoes similar transitions, although they are significantly delayed. Interestingly, the Sand equation fails to predict this transition, being off by a factor of 10 to 15, depending on the applied current density. Furthermore, inspection of the derivatives of the potential transients, $d E / d t$, not shown here, suggests the presence of two separate processes in regime III for the more intense current 
pulses. Thus, it seems reasonable to think regime III is triggered by electrochemical processes different than simple $\mathrm{Ni}^{2+}$ depletion. Finally, regime IV shows a monotonic decay of the electrode potential, which has a strong dependency on the intensity of the applied current pulse. For regimes I to III the presence of chlorides in the plating solution shift the electrode potential towards higher values, which is to be expected since the dominant species, $\mathrm{NiCl}^{+}$, has a lower reduction potential [26]. However, this difference is less noticeable for regime IV. A detailed discussion on nickel electrodeposition is beyond the scope of this work, and will be addressed in an upcoming article; however, it is important to bear all these facts in mind to interpret the results from the electrocatalytic activity experiments. SEM analysis of the Au/quartz electrodes show significant differences between the deposits generated in sulfate and chloride baths, see Figure 3. The Ni deposit generated in the sulfate bath exhibit of two types of surfaces. EDX analysis revealed areas free of deposit as well as patches of $\mathrm{Ni}$, indicating the deposition of a thin discontinuous layer. When the deposition experiment was carried out in the chloride plating bath, a uniform Ni layer was detected on gold, as confirmed by the constancy in the signal observed in the EDX analysis. Representative results of EDX elemental analysis are shown in Table 1.
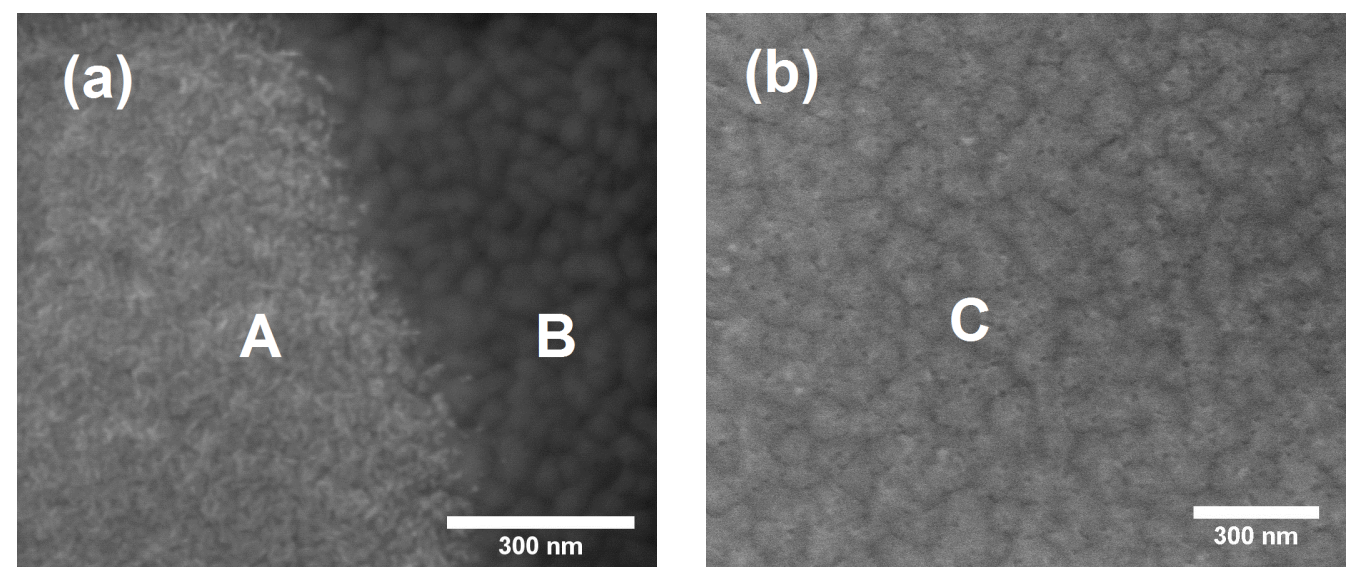

Figure 3: SEM images of $\mathrm{Ni}$ deposited on a quartz/Au electrode at $3.5 \mathrm{~mA} \mathrm{~cm}{ }^{-2}$ for five seconds. (a) $50 \mathrm{mM} \mathrm{NiSO}_{4}+500 \mathrm{mM} \mathrm{Na}_{2} \mathrm{SO}_{4}$ (b) $50 \mathrm{mM} \mathrm{NiSO}_{4}+500 \mathrm{mM} \mathrm{NaCl}$. A, B and C denote surfaces with different chemical composition as presented in Table 1.

These results agree with the bi-dimensional growth of nickel on reconstructed $\mathrm{Au}(111)$ under potentiostatic control reported by Lachenwitzer et al. $[20,27]$, and the thin layers obtained by Vampaemel et al. $[23,24]$ in absence 


\begin{tabular}{ccc}
\hline Spot & Atomic \% Au & Atomic \% Ni \\
\hline A & 87.51 & 14.29 \\
B & 100.00 & 0.00 \\
C & 95.59 & 7.41 \\
\hline
\end{tabular}

Table 1: EDX analysis of spots A, B, and C in Figure 3

of boric acid. It may be that ITO's lower wettability compared to gold, favors the formation of particles instead of thin films, as reported by Wang et al. [25].

Once the working electrode with the nickel deposit is transferred to $1 \mathrm{MKOH}, \mathrm{Ni}(\mathrm{OH})_{2}$ is formed. This is electrochemically confirmed by the presence of a surface oxidation process corresponding to the formation of $\mathrm{NiOOH}$. Curve (a) in Figure 4 corresponds a voltammogram (the last of thirty cycles) obtained in this electrolyte. Peaks $\mathrm{I}_{\mathrm{af}}$ and $\mathrm{I}_{\mathrm{cb}}$ correspond to a surface redox process by which $\mathrm{NiOOH}$ is formed [28, 29] according to the reaction [30]:

$$
\mathrm{Ni}(\mathrm{OH})_{2}+\mathrm{OH}^{-} \rightleftharpoons \mathrm{NiOOH}+\mathrm{H}_{2} \mathrm{O}+\mathrm{e}^{-}
$$

As mentioned in the previous section, repeated cycling in $\mathrm{KOH}$ was performed to improve the catalytic activity of the $\mathrm{Ni}$ film by increasing the surface concentration of the $\mathrm{NiOOH}$ by a thickening of the $\mathrm{NiOOH}$ layer $[29,31]$ and the exposure of the underlying metal by repeated lattice contraction and expansion from oxidation and reduction [32], as evidenced by the gradual increase of the area under the oxidation peak $\mathrm{I}_{\mathrm{af}}$. Curve (b) in Figure 4 is the last of thirty cycles obtained in $1 \mathrm{M} \mathrm{KOH}+0.5 \mathrm{M}$ methanol; interestingly, the $\mathrm{Ni}(\mathrm{II}) / \mathrm{Ni}(\mathrm{III})$ peaks are still present, although their potential is anodically shifted, probably due to methanol adsorption [29]. Furthermore, I $\mathrm{af}_{\mathrm{a}}$ 's peak current is significantly larger than the one observed in the last activation cycle, which indicates a further increase of the $\mathrm{NiOOH}$ surface concentration, despite the presence of methanol. The charge under peaks $\mathrm{I}_{\mathrm{af}}$ and $\mathrm{I}_{\mathrm{cb}}$ after the cycling in methanol is around 1.5 times larger than after the previous activation cycling in $\mathrm{KOH}$.

At higher potentials, two anodic peaks, one in the forward and one in the backward scan, appear. These peaks, $\mathrm{II}_{\mathrm{af}}$ and $\mathrm{II}_{\mathrm{ab}}$, correspond to methanol oxidation on fresh $\mathrm{NiOOH}$ and reactivated $\mathrm{NiOOH}$ by desorption of poisonous species, respectively [33]. Contrary to what was reported by Rahim et al. [28], the maximum current of these peaks also increases with the number of cycles, indicating that the morphology of the $\mathrm{Ni}$ deposits obtained in this work have better stability against the loss of activity. The potential difference between 


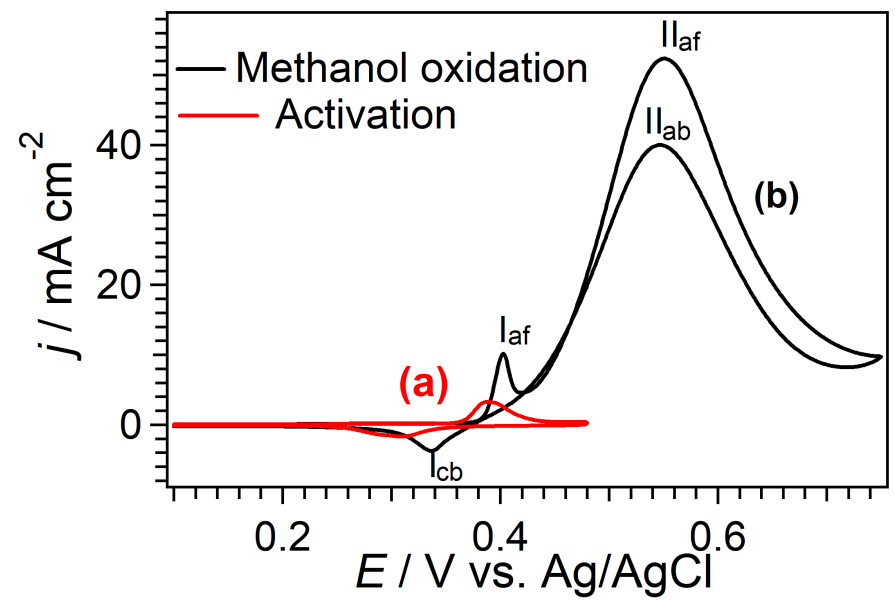

Figure 4: Typical cyclic voltammogram of electrodeposited $\mathrm{Ni}$ films at $50 \mathrm{mV} \mathrm{s}^{-1}$ in (a) $1 \mathrm{M} \mathrm{KOH}$ and (b) $1 \mathrm{M} \mathrm{KOH}+0.5 \mathrm{M}$ methanol.

$\mathrm{I}_{\mathrm{af}}$ and $\mathrm{I}_{\mathrm{bc}}, \Delta E_{p}$, serves as an indicator of the kinetics of the electron transfer [34]. Chen et al. [29] and Casella et al. [35] found a clear influence of the $\mathrm{NiOOH}$ thickness on $\Delta E_{p}$; thicker layers would result in larger $\Delta E_{p}$ values, ranging from $84 \pm 9 \mathrm{mV}$ to $199 \pm 23 \mathrm{mV}$, which implies slower kinetics. For the electrodeposited nickel films of this work, $\Delta E_{p}$ appears to be independent of the applied current pulse; the average values were $62 \pm 5 \mathrm{mV} \mathrm{mV}$ for the sulfate bath, and $55 \pm 5 \mathrm{mV}$ for the chloride bath. This suggests that regardless of the applied current, the resulting film is thin enough to allow a reversible electron transfer.

The surface concentration of $\mathrm{NiOOH}, \Gamma$, was estimated using the following equation [36]:

$$
\Gamma=\frac{Q}{n F A}
$$

where $Q$ is the charge under peak $\mathrm{I}_{\mathrm{af}}, F$ is Faraday's constant, $n$ the number of electrons transferred during the redox process, and $A$ is the geometric area of the working electrode. The effectiveness of the nickel deposits to oxidize methanol was evaluated though their electrocatalytic intensity $(E I)$. This parameter was introduced by Ding et al. [37]; it is defined as the sum of $\mathrm{II}_{\mathrm{af}}$ and $\mathrm{II}_{\mathrm{ab}}$ peak currents. Both $\Gamma$ and $E I$ were taken from the last voltammogram obtained in the presence of $0.5 \mathrm{M}$ methanol.

Figures 5a and 5b show the dependence of $\Gamma$ and $E I$ (left and right axis) on the applied deposition current for the sulfate and chloride baths, respectively. It is interesting to see that in both electrolytes $\Gamma$ reaches a maximum value close to $4 \mathrm{~mA} \mathrm{~cm}^{-2}$; however, the surface concentrations, $\Gamma$, obtained for the 

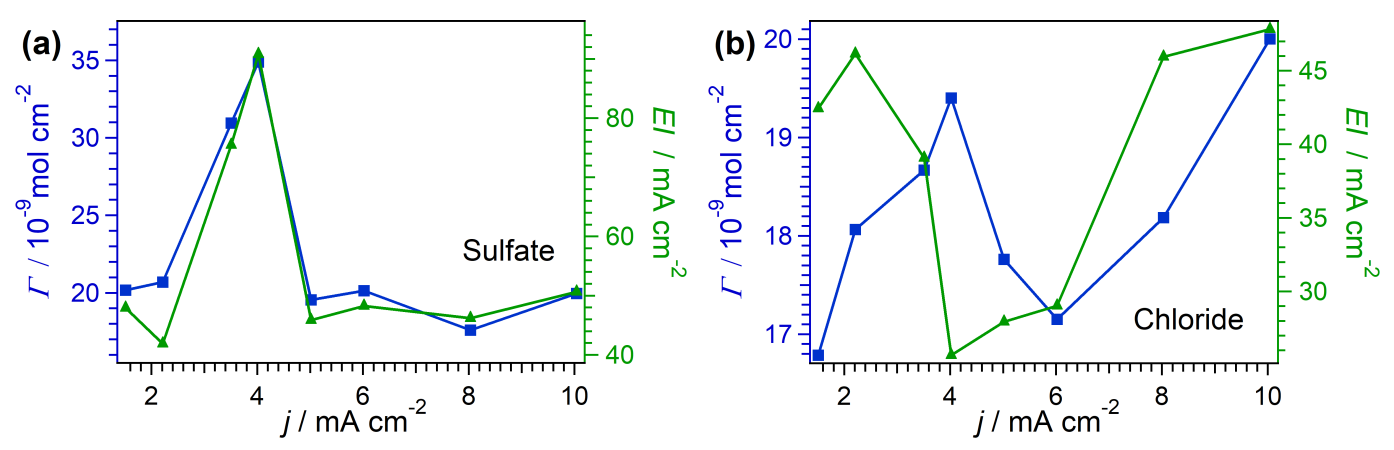

Figure 5: Dependence of $\Gamma$ (left blue axis) and $E I$ (right green axis) on the $\mathrm{Ni}$ electrodeposition current density for (a) sulfate bath and (b) chloride bath.

deposits from the sulfate bath were consistently larger than those from the chloride bath. This difference is also observed for the $E I$ values, i.e., the sulfate bath deposits had significantly larger activity for methanol oxidation than their chloride counterparts. Interestingly, the $E I$ behavior of the sulfate deposits is mirrored almost perfectly by the behavior of $\Gamma$, indicating a strong dependence of the catalytic activity on the surface concentration of $\mathrm{NiOOH}$. In contrast, the chloride deposits do not show the same correspondence between $E I$ and $\Gamma$. The voltammograms in Figure 4, and the EI data in Figure 5, were normalized with respect to the working electrode geometric area in order to make comparisons with other works. The activity of the sulfate deposits is similar, and in some cases even larger, than those reported under comparable conditions (see Table 2).

\begin{tabular}{lccc}
\hline Synthesis & Scan Rate $/ \mathrm{mV} \mathrm{s}^{-1}$ & $E I / \mathrm{mA} \mathrm{cm}^{-2}$ & Reference \\
\hline $\begin{array}{l}\text { Galvanostatic } \\
\text { electrodeposition }\end{array}$ & 50 & 90 & This work \\
$\begin{array}{l}\text { Atomic layer } \\
\text { deposition }\end{array}$ & 50 & 20 & $\begin{array}{c}\text { Tong et al. } \\
{[15]}\end{array}$ \\
$\begin{array}{l}\text { Ion implantation } \\
\text { Electroless }\end{array}$ & 50 & 8.5 & $\begin{array}{c}\text { Guo et al. } \\
\text { deposition }\end{array}$ \\
\hline
\end{tabular}

Table 2: Comparison between the values of $E I$ obtained in this work and other with similar experimental conditions. $0.5 \mathrm{M}$ methanol in $1 \mathrm{M} \mathrm{KOH}$. 
Rahim et al. [28] evaluated the catalytic activity of their galvanostatically generated deposits through potentiostatic polarization in $0.5 \mathrm{M}$ methanol + $1 \mathrm{M} \mathrm{KOH}$. Their deposits exhibit similar activity to the ones of this work, even though the deposition times used by them are two orders of magnitude longer. The larger activity of the electrodeposited Ni thin films may be explained by the work of Liao et al. [38]. They found that the electrocatalytic activity of $\mathrm{Au}(111)$ supported $\mathrm{Pt}$ monolayer films towardss hydrogen oxidation and evolution was larger than the electrocatalytic activity of $\operatorname{Pt}(111)$. This was attributed to a larger interatomic spacing of the Pt monolayer film. This phenomenon may well be extended to other metal monolayers and their oxides, as the $\mathrm{Ni}$ deposits and resulting $\mathrm{NiOOH}$ presented in this work. Nonetheless, it cannot be ignored the fact that the current measured during an electrocatalyzed process is an extensive quantity, dependent on the electrocatalyst surface area; thus, in order to evaluate the activity of a thin film or of a highly porous material a precise knowledge of the active area is required. Therefore, normalization to the effective surface area, $\Gamma$, would allow a better understanding of the intrinsic electrocatalytic properties of the produced $\mathrm{NiOOH}$ films.
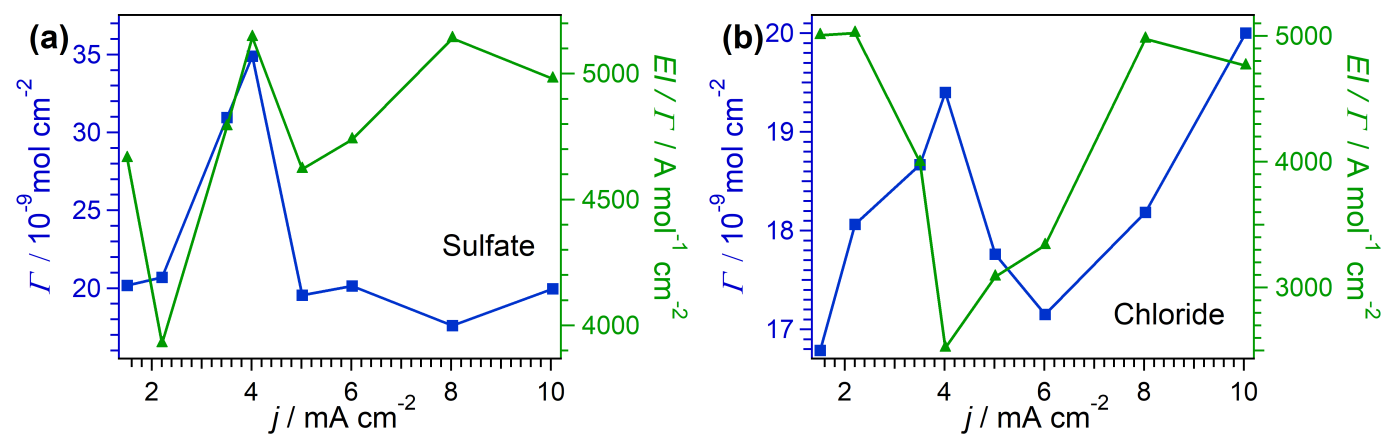

Figure 6: Dependence of $\Gamma$ (left blue axis) and $E I / \Gamma$ (right green axis) on the deposition current. (a) sulfate bath (b) chloride bath.

Although the exact surface area of the nickel deposits is unknown and cannot be measured in a simple, non-destructive manner, EI can be normalized to $\Gamma$ since it reflects the surface concentration the electrocatalytic species. The dependence of the resulting quantity $E I / \Gamma$ with respect to the applied current density for the sulfate deposits is shown in Fig. 6a. While the film obtained by applying $4.0 \mathrm{~mA} \mathrm{~cm}{ }^{-2}$ remains the best catalyst, the $8.0 \mathrm{~mA} \mathrm{~cm}^{-2}$ film shows a comparable electrocatalytic activity.

Moreover, when comparing the $E I / \Gamma$ values between the $4.0 \mathrm{~mA} \mathrm{~cm}^{-2}$ film and other current densities, except for the $2.2 \mathrm{~mA} \mathrm{~cm}^{-2}$, is significantly 
smaller than for the non-normalized data. This results in the dependencies of $E I / \Gamma$ and $E I$ on the deposition current density being qualitatively different. On the contrary, the $E I / \Gamma$ data for chloride deposits show more or less the same dependency on the deposition current and the non-normalized $E I$, as depicted in Figure 6b. These results indicate that the catalytic activity of the chloride deposits is determined mainly by the intrinsic properties of the nickel film, while for the sulfate deposits the intrinsic catalytic activity is significantly increased by a large surface concentration of $\mathrm{NiOOH}$.

\section{Conclusions}

Nickel films active towards the electrocatalytic oxidation of methanol were electrodeposited on gold though galvanostatic control in a borate-free electrolyte. Even with the short deposition time of two seconds the resulting materials in alkaline medium showed good catalytic activity for methanol electrooxidation in alkaline media. After 30 cycles the nickel films did not show any degradation. The nature of the anion in the supporting electrolyte had a strong effect on the $\mathrm{NiOOH}$ surface concentration, $\Gamma$ and showed a strong correlation with the electrocatalytic activity; sulfates lead to the production of the most active materials, in particular those obtained with a current density pulse of $4.0 \mathrm{~mA} \mathrm{~cm}{ }^{-2}$ showed the best activity, while the presence of chlorides caused significantly lower values of $\Gamma$, indicating that this can be used as an important performance parameter for the optimization of anode electrodes in DAFCs.

Acknowledgements The authors express their gratitude to CONACyT for the postdoctoral fellowship granted to DACY through the CONACYT-SENER 20162017 and to the School of Engineerin and Sciences for the financial support received for the development of this research through the Focus Group of Optics and Lasers.

\section{References}

[1] R. Dillon, S. Srinivasan, A.S. Arció, and V. Antonucci, "International activities in DMFC R \& D: status of technologies and potential applications", Journal of Power Sources 127(1), pp. 112 - 126 (2004), Eighth Ulmer Electrochemische Tage. 
[2] Kenneth Ikechukwu Ozoemena, "Nanostructured platinum-free electrocatalysts in alkaline direct alcohol fuel cells: catalyst design, principles and applications", $R S C A d v$. 6, pp. 89523-89550 (2016).

[3] F. Ahmad Zakil, S.K. Kamarudin, and S. Basri, "Modified nafion membranes for direct alcohol fuel cells: An overview", Renewable and Sustainable Energy Reviews 65, pp. 841 - 852 (2016).

[4] B.B. Blizanac, P.N. Ross, and N.M. Markovic, "Oxygen electroreduction on ag(111): The ph effect", Electrochimica Acta 52(6), pp. 2264 - 2271 (2007),

[5] A.V Tripković, K.D Popović, B.N Grgur, B Blizanac, P.N Ross, and N.M Marković, "Methanol electrooxidation on supported pt and ptru catalysts in acid and alkaline solutions", Electrochimica Acta 47(22), pp. 3707 3714 (2002).

[6] J. Prabhuram and R. Manoharan, "Investigation of methanol oxidation on unsupported platinum electrodes in strong alkali and strong acid", Journal of Power Sources 74(1), pp. 54 - 61 (1998).

[7] Sofía Carugno, Elisabeth Chassaing, Michel Rosso, and Graciela A. González, "Enhanced electrochemical oxidation of methanol on copper electrodes modified by electrocorrosion and electrodeposition", Materials Chemistry and Physics 143(3), pp. 1012 - 1017 (2014).

[8] F. Bidault, D.J.L. Brett, P.H. Middleton, and N.P. Brandon, "Review of gas diffusion cathodes for alkaline fuel cells", Journal of Power Sources $\mathbf{1 8 7}(1)$, pp. $39-48$ (2009).

[9] M. Fleischmann, K. Korinek, and D. Pletcher, "The kinetics and mechanism of the oxidation of amines and alcohols at oxide-covered nickel, silver, copper, and cobalt electrodes", J. Chem. Soc., Perkin Trans. 2, pp. 1396-1403 (1972).

[10] Derek Pletcher, "Electrocatalysis: present and future", Journal of Applied Electrochemistry 14(4), pp. 403-415 (1984).

[11] Marcio Vidotti, Carolina D. Cerri, Rafaela F. Carvalhal, Jailson C. Dias, Renata K. Mendes, Susana I. Córdoba de Torresi, and Lauro T. Kubota, "Nickel hydroxide electrodes as amperometric detectors for carbohydrates in flow injection analysis and liquid chromatography", Journal of Electroanalytical Chemistry 636(1), pp. 18 - 23 (2009). 
[12] Juan Wang, Qi Zhao, Hongshuai Hou, Yifei Wu, Weizhen Yu, Xiaobo Ji, and Lidong Shao, "Nickel nanoparticles supported on nitrogen-doped honeycomb-like carbon frameworks for effective methanol oxidation", RSC Adv. 7, pp. 14152-14158 (2017).

[13] Su-Juan Li, Wei Guo, Bai-Qing Yuan, Dao-Jun Zhang, Zhu-Qing Feng, and Ji-Min Du, "Assembly of ultrathin niooh nanosheets on electrochemically pretreated glassy carbon electrode for electrocatalytic oxidation of glucose and methanol", Sensors and Actuators B: Chemical 240, pp. 398 - 407 (2017).

[14] Qingfeng Yi, Wu Huang, Jingjing Zhang, Xiaoping Liu, and Lei Li, "Methanol oxidation on titanium-supported nano-scale ni flakes", Catalysis Communications 9(10), pp. 2053 - 2058 (2008).

[15] Xili Tong, Yong Qin, Xiangyun Guo, Oussama Moutanabbir, Xianyu Ao, Eckhard Pippel, Lianbing Zhang, and Mato Knez, "Enhanced catalytic activity for methanol electro-oxidation of uniformly dispersed nickel oxide nanoparticles - carbon nanotube hybrid materials", Small 8(22), pp. 3390-3395 (2012).

[16] Meisong Guo, Yanan Yu, and Jingbo Hu, "Nickel nanoparticles for the efficient electrocatalytic oxidation of methanol in an alkaline medium", Electrocatalysis 8(4), pp. 392-398 (2017).

[17] Gh. S. Ferdowsi, S. A. Seyedsadjadi, and A. Ghaffarinejad, "Ni nanoparticle modified graphite electrode for methanol electrocatalytic oxidation in alkaline media", Journal of Nanostructure in Chemistry 5(1), pp. 17-23 (2015).

[18] Gerardo T. Martínez, Genaro Zavala, and Marcelo Videa, "Electrodeposition of Nickel Particles and their Characterization", Journal of the Mexican Chemical Society 53, pp. 7 - 11 (2009).

[19] M. Łukaszewski, M. Soszko, and A. Czerwiński1, "Electrochemical methods of real surface area determination of noble metal electrodesan overview", International Journal of Electrochemical Science 11, pp. 4442-4469 (2006).

[20] A. Lachenwitzer and O. M. Magnussen, "Electrochemical quartz crystal microbalance study on the kinetics of nickel monolayer and multilayer electrodeposition on (111)-oriented gold films", The Journal of Physical Chemistry B 104(31), pp. 7424-7430 (2000). 
[21] Alexander Milchev and M. Irene Montenegro, "A galvanostatic study of electrochemical nucleation", Journal of Electroanalytical Chemistry 333(1), pp. 93 - 102 (1992),

[22] Vladimir A. Isaev and Olga V. Grishenkova, "Galvanostatic phase formation", Journal of Solid State Electrochemistry 18(9), pp. 2383-2386 (2014).

[23] Johannes Vanpaemel, Marleen H. van der Veen, Stefan De Gendt, and Philippe M. Vereecken, "Enhanced nucleation of ni nanoparticles on tin through h3bo3-mediated growth inhibition", Electrochimica Acta 109, pp. $411-418$ (2013).

[24] Johannes Vanpaemel, Masahito Sugiura, Daniel Cuypers, Marleen H. van der Veen, Stefan De Gendt, and Philippe M. Vereecken, "Electrochemical deposition of subnanometer ni films on tin", Langmuir 30(8), pp. 2047-2053 (2014), PMID: 24520857.

[25] Rongyue Wang, Ugo Bertocci, Haiyan Tan, Leonid A. Bendersky, and Thomas P. Moffat, "Self-terminated electrodeposition of ni, co, and fe ultrathin films", The Journal of Physical Chemistry C 120(29), pp. 1622816237 (2016).

[26] M. Peykova, E. Michailova, Stoychev, and A. Milchev, "Galvanostatic studies of the nucleation and growth kinetics of copper in the presence of surfactants", Electrochimica Acta 40(16), pp. 2595 - 2601 (1995).

[27] F. A. Möller, J. Kintrup, A. Lachenwitzer, O. M. Magnussen, and R. J. Behm, "In situ stm study of the electrodeposition and anodic dissolution of ultrathin epitaxial ni films on au(111)", Phys. Rev. B 56, pp. 1250612518 (1997).

[28] M.A. Abdel Rahim, R.M. Abdel Hameed, and M.W. Khalil, "Nickel as a catalyst for the electro-oxidation of methanol in alkaline medium", Journal of Power Sources 134(2), pp. 160 - 169 (2004).

[29] Dayi Chen and Shelley D. Minteer, "Mechanistic study of nickel based catalysts for oxygen evolution and methanol oxidation in alkaline medium", Journal of Power Sources 284, pp. 27 - 37 (2015).

[30] M. Fleischmann, K. Korinek, and D. Pletcher, "The oxidation of organic compounds at a nickel anode in alkaline solution", Journal of Electroanalytical Chemistry and Interfacial Electrochemistry 31(1), pp. 39 - 49 (1971). 
[31] M. E. G. Lyons, R. L. Doyle, I. Godwin, M. O'Brien, and L. Russell, "Hydrous nickel oxide: Redox switching and the oxygen evolution reaction in aqueous alkaline solution", Journal of The Electrochemical Society 159(12), pp. H932-H944 (2012).

[32] David S. Hall, Christina Bock, and Barry R. MacDougall, "The electrochemistry of metallic nickel: Oxides, hydroxides, hydrides and alkaline hydrogen evolution", Journal of The Electrochemical Society 160(3), pp. F235-F243 (2013).

[33] I. Danaee, M. Jafarian, F. Forouzandeh, F. Gobal, and M.G. Mahjani, "Electrocatalytic oxidation of methanol on ni and nicu alloy modified glassy carbon electrode", International Journal of Hydrogen Energy 33(16), pp. 4367 - 4376 (2008).

[34] Aleksander Ciszewski and Grzegorz Milczarek, "Electrocatalytic oxidation of alcohols on glassy carbon electrodes electrochemically modified by conductive polymeric nickel(ii) tetrakis(3-methoxy-4-hydroxyphenyl) porphyrin film", Journal of Electroanalytical Chemistry 413(1), pp. 137 - 142 (1996).

[35] Innocenzo G. Casella and Maria Gatta, "Electrocatalysis and detection of carbohydrates byanion-exchange chromatography at a gold substrate electrode modified with nickel cyanide ions", Electroanalysis 13(7), pp. 549-554 (2001).

[36] S.M. Golabi and A. Nozad, "Electrocatalytic oxidation of methanol on a nickel-porphyrin ix complex modified glassy carbon electrode in alkaline medium", Electroanalysis 16(3), pp. 199-209 (2004).

[37] Keqiang Ding, Yongbo Zhao, Likun Liu, Yanli Cao, Qingfei Wang, Hongbo Gu, Xingru Yan, and Zhanhu Guo, "PtNi bimetallic composite nanocatalysts prepared by using multi-walled carbon nanotubes as reductants for ethanol oxidation reaction", International Journal of Hydrogen Energy 39(31), pp. 17622 - 17633 (2014).

[38] Weicheng Liao and Shuehlin Yau, "Au(111)-supported pt monolayer as the most active electrocatalyst toward hydrogen oxidation and evolution reactions in sulfuric acid", The Journal of Physical Chemistry C 121(35), pp. 19218-19225 (2017). 